EPSC Abstracts

Vol.14, EPSC2020-1036, 2020

Europlanet Science Congress 2020

(C) Author(s) 2020. This work is distributed under

the Creative Commons Attribution 4.0 License.

\title{
Neutral Aromatic Pathways Enhanced by EUV Irradiation in a Titan's Atmosphere Simulation Photochemical Experiment
}

\author{
Jérémy Bourgalais ${ }^{1,2}$, Nathalie Carrasco ${ }^{2}$, Ludovic Vettier ${ }^{2}$, Antoine Comby $^{3}$, Valérie Blanchet ${ }^{3}$, \\ Jérôme Gaudin ${ }^{3}$, Yann Mairesse ${ }^{3}$, and Bernard Marty ${ }^{1}$ \\ ${ }^{1}$ Centre de Recherches Pétrographiques et Géochimiques, UMR 7358 CNRS-Université de Lorraine, 15 rue Notre Dame des \\ Pauvres, BP 20, F-54501 Vandoeuvre-lès-Nancy, France \\ ${ }^{2}$ LATMOS-IPSL, Université Versailles St-Quentin, CNRS/INSU, Sorbonne Université, UPMC Univ. Paris 06,11 boulevard \\ d'Alembert, 78280 Guyancourt, France (jeremy.bourgalais@latmos.ipsl.fr) \\ ${ }^{3}$ CELIA, Université de Bordeaux - CNRS - CEA, UMR5107, 351 Cours de la Libération F33405 Talence, France
}

\begin{abstract}
In the atmosphere of Titan, Saturn's main satellite, molecular growth is initiated by a chemistry involving charged and free-radical species. However, the respective contribution of these species to the complexification of matter is far from being known. This work presents a chemical analysis by mass spectrometry at relatively low pressure to characterize the formation pathways of the first aromatics, notably benzene, by irradiating a mixture $\mathrm{N}_{2} / \mathrm{CH}_{4}$ with EUV photons.
\end{abstract}

\section{Introduction}

It is now accepted that photochemical processes in the atmosphere of Titan, the largest of Saturn's 62 moons, directly influence the chemical composition of the liquid lakes and the surface hydrological activity. Thus, a detailed understanding of the chemistry of Titan's atmosphere is the way forward to a whole Titan modeling. In the highest atmospheric layers between 700 and 1100 $\mathrm{km}$ in altitude, $\mathrm{N}_{2}$ and $\mathrm{CH}_{4}$ are ionized and dissociated mainly by energetic EUV photons from solar radiation, leading to the formation of reactive species like ions and radicals among a diverse range of species hydrocarbons, amines, and imines. In the lower layers of the atmosphere, below 500km, hydrocarbons such as benzene and other aromatics by interaction with lower energy UV photons (>155 nm) are key compounds in the formation of larger and more complex molecules like Polycyclic Aromatic Hydrocarbons (PAHs) and Polycyclic Aromatic Nitrogen Heterocycles (PANHs). These intermediate species between small organic precursor molecules and larger carbonaceous materials, contribute to the production of aerosols which form the opaque photochemical haze layers that obscure the surface of Titan. Thus the identification of the pathways of formation of these species is essential for understanding the chemistry of Titan's atmosphere, but is yet to be fully understood even for the simplest $\mathrm{PAH}$, namely benzene $\left(\mathrm{C}_{6} \mathrm{H}_{6}\right)$. In situ and direct observations are insufficient to constrain photochemical models and understand the molecular complexification pathways of Titan's atmosphere. Thus the way forward is to reproduce the chemistry in the laboratory by irradiating a mixture of gases representative of this environment from a photonproducing energy source. 


\section{Experimental Method}

In this study a nonlinear optics approach has been used to generate intense and stable light in the Extreme Ultraviolet (EUV) because energetic photons are absorbed in the upper atmospheric layers and initiate the formation of reactive species in the satellite ionosphere. The EUV light was produced by the high-order harmonic generation of the femtosecond BLASBEAT laser at CELIA in Bordeaux and injected into the closed and removable SURFACAT photoreactor to irradiate for several hours, at a pressure of $0.1 \mathrm{mbar}$, a gas mixture composed of nitrogen and methane $(95 / 5 \%)$ at a wavelength of $85.58 \mathrm{~nm}$. This wavelength falls within the energy range of interest between the $\mathrm{CH}_{4}$ dissociation threshold $(98 \mathrm{~nm})$ and the $\mathrm{N}_{2}$ ionization threshold $(79.4 \mathrm{~nm})$, which allows the production of nitrogen atoms in its ground and excited state that are suspected of playing a fundamental role in the molecular growth of complex but as yet unknown nitrogen species.

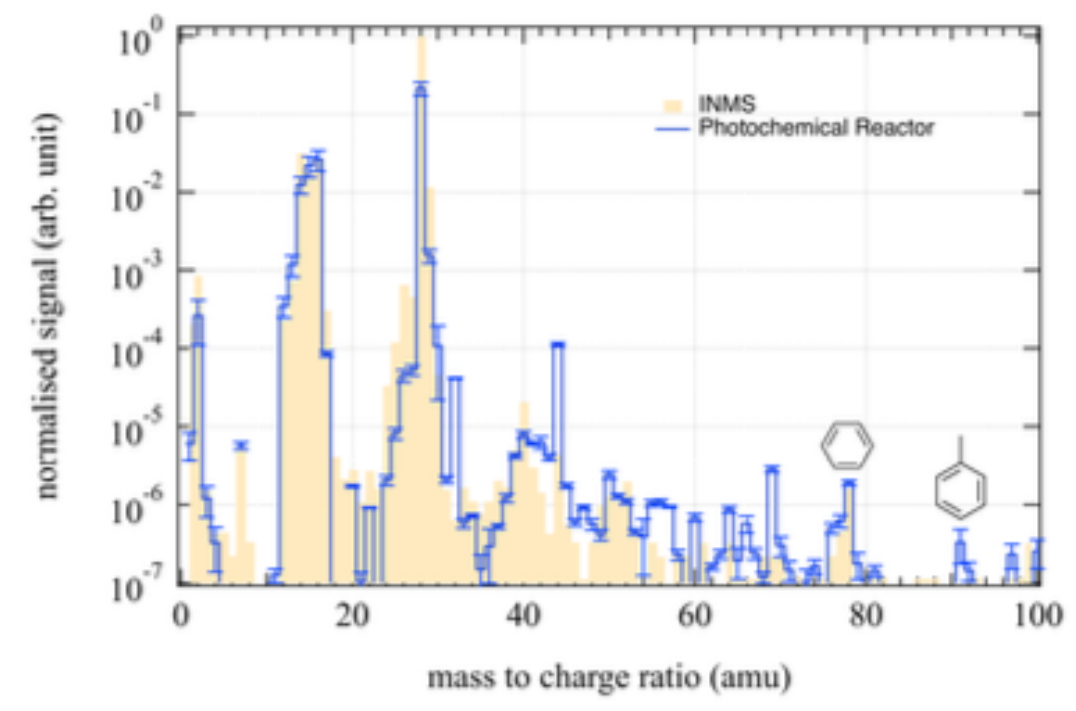

Figure 1: Background corrected mass spectrum obtained after 3 hours at $85.58 \mathrm{~nm}$ compared to the average mass spectrum from 1 to 99 atomic mass unit measured by Ion Neutral Mass Spectrometer (INMS) between the altitudes of 1174 and $1230 \mathrm{~km}$. Data are normalized to peak mass 16 .

\section{Results}

The results presented in Figure 1, show overall a good agreement between the products formed in the photochemical reactor and the data obtained in situ in Titan's atmosphere. Especially, there is good agreement for the peak at mass 78 which has been identified as benzene in Titan's atmosphere. Because of the importance of benzene in Titan's atmosphere, this study is dedicated to analyze its formation pathways in the photochemical reactor and the implications for our understanding of the chemistry of Titan's atmosphere. At the wavelength used in this experiment, 
photolysis of methane leads mainly to the formation of the $\mathrm{CH}_{4}{ }^{+}$ion with a branching ratio of $47 \%$ and methyl radical ion $\left(\mathrm{CH}_{3}{ }^{+}\right)$and methylidyne radical $(\mathrm{CH})$ with branching ratios of $19 \%$ and $18 \%$ respectively. While the formation of $\mathrm{CH}_{4}{ }^{+}$leads to a closed cycle of methane formation, $\mathrm{CH}_{3}{ }^{+}$is a source of $\mathrm{C}_{2} \mathrm{H}_{5}{ }^{+}$ions by reaction with methane. These ions recombine to lead mainly to the formation of highly reactive ethylenyl radicals $\left(\mathrm{C}_{2} \mathrm{H}_{3}\right)$. By reacting with hydrocarbon radicals such as butadienyl $\left(\mathrm{C}_{4} \mathrm{H}_{3}\right)$, they are at the origin of phenyl radicals $\left(\mathrm{C}_{6} \mathrm{H}_{5}\right)$, precursor of benzene by addition of a hydrogen atom. At the wavelength used in this experiment, the other dominant photoproduct of methane photolysis, $\mathrm{CH}$, leads mainly to the formation of $\mathrm{C}_{2} \mathrm{H}_{4}$, whose photolysis by energetic photons leads mainly to the formation of hydrocarbon ions $\mathrm{C}_{2} \mathrm{H}_{2}{ }^{+}$and $\mathrm{C}_{2} \mathrm{H}_{3}{ }^{+}$. These ions then lead to the formation of propargyl radicals $\left(\mathrm{C}_{3} \mathrm{H}_{3}\right)$ which by recombining give rise to the formation of benzene.

\section{Implications for Titan's atmosphere}

The literature points out that the production of benzene in the upper atmospheric layers of Titan is due to radical-radical reactions but mainly to the chemistry of the ions via the dissociative recombination of aromatic ions such as $\mathrm{C}_{6} \mathrm{H}_{6}{ }^{+}$and $\mathrm{C}_{6} \mathrm{H}_{7}{ }^{+}$. In less energetic conditions, such as photons from solar radiation arriving on Titan, the photolysis of methane leads mainly to the formation of hydrocarbon radicals $\left(\mathrm{CH}_{2}\right.$ and $\left.\mathrm{CH}_{3}\right)$ which will contribute to many small neutral hydrocarbons $\left(\mathrm{C}_{2} \mathrm{H}_{2}, \mathrm{C}_{2} \mathrm{H}_{4}\right)$ that will form heavy ions and prevent electronic recombinations. However, the troubling overlap between the benzene signal in this EUV-dominated work and that of INMS shows that it is crucial to have a good assessment of the branching ratios of radical-radical reactions at low pressures and low temperatures representative of Titan's atmosphere. Especially, association reactions can be rapid even at low pressures for adducts of significant size. Indeed, the most energetic photons are absorbed in the upper atmospheric layers to initiate molecular growth while the less energetic photons are absorbed lower in the atmosphere. Additionally, the detection of a signal at mass 92 in Figure 1, could be assigned to toluene $\left(\mathrm{C}_{7} \mathrm{H}_{8}\right)$ and signal at mass 91 to its main fragment due to electronic impact by the ionization of the mass spectrometer used in this work. It confirms the importance of a rigorous analysis of neutral reactions, since toluene can be formed by the reaction between phenyl and methyl radicals, which, although poorly understood, involves one of the precursors of benzene whose formation would be favored in an environment with energetic photons. 